\title{
Cavity enhancement of the magneto-optic Kerr effect for optical studies of magnetic nanostructures
}

Aaron R. Hawkins

hawkins@ee.byu.edu

N. Qureshi

Holger Schmidt

Follow this and additional works at: https://scholarsarchive.byu.edu/facpub

Part of the Electrical and Computer Engineering Commons

\section{Original Publication Citation}

Qureshi, N., H. Schmidt, and A. R. Hawkins. "Cavity enhancement of the magneto-optic Kerr effect for optical studies of magnetic nanostructures." Applied Physics Letters 85 (24): 431-433

\section{BYU ScholarsArchive Citation}

Hawkins, Aaron R.; Qureshi, N.; and Schmidt, Holger, "Cavity enhancement of the magneto-optic Kerr effect for optical studies of magnetic nanostructures" (2004). Faculty Publications. 424.

https://scholarsarchive.byu.edu/facpub/424 


\title{
Cavity enhancement of the magneto-optic Kerr effect for optical studies of magnetic nanostructures
}

\author{
N. Qureshi ${ }^{\mathrm{a}}$ and H. Schmidt \\ School of Engineering, University of California, 1156 High Street, Santa Cruz, California 95064 \\ A. R. Hawkins \\ ECE Department, Brigham Young University, 459 Clyde Building, Provo, Utah 84602
}

(Received 6 February 2004; accepted 24 May 2004)

\begin{abstract}
We present a study of cavity enhancement of the magneto-optic Kerr effect using dielectric multilayers in order to facilitate optical studies of individual single-domain nanomagnets. We develop a transfer matrix theory to analyze Kerr rotation from an arbitrary number of possibly lossy dielectric layers. The combination of one lossless and one thin metallic layer is found to be most favorable for studying individual nanomagnets, providing the best tradeoff between signal enhancement and spatial resolution. Accounting for the microscopic surface structure, we find good agreement between theory and experiment. Using this technique, we demonstrate Kerr enhancements by a factor of more than 16. (C) 2004 American Institute of Physics.
\end{abstract}

[DOI: $10.1063 / 1.1774276]$

Single-domain ferromagnetic nanostructures have recently received a lot of interest, mainly due to their potential use in high-density magnetic storage media ${ }^{1}$ and spintronics. ${ }^{2}$ The advent of nanofabrication capabilities has created opportunities to study phenomena such as magnetization switching that have been theoretically investigated for a long time in near-ideal single-domain particles. ${ }^{3-5}$ Nanomagnetic structures have indeed been fabricated in a number of different ways and materials ${ }^{6,7}$ and the standard characterization method has been magnetic force microscopy (MFM) based on its relative ease and high spatial resolution. The major drawbacks of MFM are its intrinsically perturbative nature (the field from the magnetic tip itself interacts with the sample) and slow scan speed, which complicate studies of magnetization switching ${ }^{8}$ and preclude the observation of magnetization dynamics. Optical techniques based on the magneto-optic Kerr effect (MOKE) do not suffer from these problems. ${ }^{9}$ In order to spatially resolve individual singledomain magnets with dimensions on the order of $100 \mathrm{~nm}$, near-field optical scanning techniques have been used successfully. ${ }^{10}$ The difficulties lie in simultaneously achieving spatial resolution and sufficient signal-to-noise ratio because polarization rotations due to magnetization reversal are small and fiber tips with small apertures reduce polarization extinction and light intensity. ${ }^{11}$

It has been known for some time that the polarization rotation due to MOKE from a magnetic surface can be increased by depositing dielectric films on the magnetic surface. ${ }^{12-14}$ This technique is promising for nanomagnetics studies since it is compatible with both nanofabrication and near-field measurements. The MOKE signal enhancement is due to a cavity effect where the dielectric layer acts as a Fabry-Perot etalon that gives rise to multiple reflections off the magnetic layer. If the layer thicknesses are chosen correctly, these partial reflections add up in phase and the polarization rotation of the total reflected field is increased. MOKE enhancement up to a factor of 4 has been observed using a single dielectric film ${ }^{12,14}$ on a reflective magnetic material. The agreement between theory and experiment for

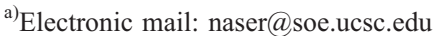

lossy materials has been rather poor and a systematic study of the possible enhancement based on the number and composition of the layers has not yet been reported.

In this letter, we systematically analyze cavity enhancement of the polar magneto-optic Kerr rotation for improving the signal-to-noise ratio of optical studies on single nanomagnets. We describe a matrix formalism that allows us to consider the situation of multiple lossy dielectric layers and identify the best solution for simultaneous Kerr enhancement and spatial resolution. The method takes into account the microscopic structure of the multilayers yielding good agreement with experimental data. In such an optimized structure, we observe the largest Kerr enhancement to date.

The experimental situation we consider is shown in Fig. 1. A magnetic surface (complex refractive index $n$ ) with magnetization perpendicular to the surface (polar Kerr effect) is coated with $k$ dielectric layers (complex indices $n_{1}, \ldots, n_{k}$, thickness $L_{1}, \ldots, L_{k}$ ). A linearly polarized beam of vacuum wavelength $\lambda$ is incident on the top layer and we assume an incident polarization $E_{i}$ in the $x$ direction. The resulting polar Kerr rotation is given by $\Phi$ $=\arctan \left[\left(\mathbf{E}_{r}\right)_{y} /\left(\mathbf{E}_{r}\right)_{x}\right]$. This problem has been solved more generally for magnetic multilayers and any incident angle using a system of $2 \times 2$ transmission matrices for each layer, ${ }^{15}$ and still more generally by formulating $4 \times 4$ boundary and propagation matrices for each layer with arbitrary magnetization direction. ${ }^{16}$ In our specialization to single nanomagnets, however, the experimental emphasis is on polar Kerr rotation and on only one reflective magnetic layer. Similar to Refs. 15 and 16, we introduce a matrix approach that describes each dielectric layer and interface by a transfer

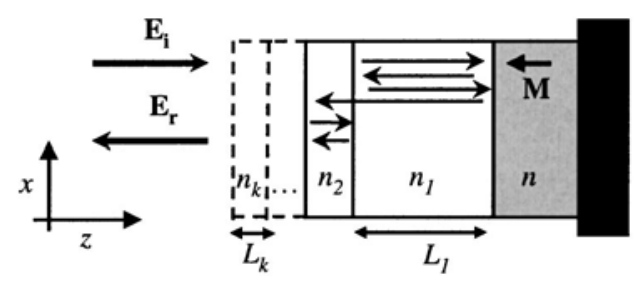

FIG. 1. A cavity formed between a magnetized structure (complex refractive index $n)$ and a nonmagnetic dielectric multilayer sequence $\left(n_{1}, n_{2}, \ldots, n_{k}\right)$. 
matrix that accounts for magneto-optic rotations, but in a form specifically adapted to this experimental situation.

To account for the polarization mixing upon reflection from the gyrotropic medium, we modify the standard T-matrix theory ${ }^{17}$ by describing the reflection coefficients $\mathbf{r}_{\mathbf{n}}$ as $2 \times 2$ matrices. At the magnet-dielectric interface, polarization rotation is a result of the differing refractive indices $r_{+}$and $r_{-}$for the two circular polarization components. ${ }^{18}$ For linear polarization components this is

$$
\mathbf{r}_{1}=\frac{1}{2}\left[\begin{array}{cc}
r_{+}+r_{-} & i\left(r_{+}-r_{-}\right) \\
-i\left(r_{+}-r_{-}\right) & r_{+}+r_{-}
\end{array}\right] \text {. }
$$

The field reflected from the multilayer structure is calculated from the total T-matrix of the system. As specific examples, the total T-matrix for the one-layer Fabry-Perot cavity is

$$
\mathbf{T}^{(1)}=\left(\mathbf{t}_{1} \mathbf{t}_{2}\right)^{-1}\left[\begin{array}{ll}
\mathbf{I} e^{i \theta}+\mathbf{r}_{1} \mathbf{r}_{2} e^{-i \theta} & \mathbf{r}_{2} e^{i \theta}+\mathbf{r}_{1} e^{-i \theta} \\
\mathbf{r}_{1} e^{i \theta}+\mathbf{r}_{2} e^{-i \theta} & \mathbf{I} e^{-i \theta}+\mathbf{r}_{1} \mathbf{r}_{2} e^{i \theta}
\end{array}\right],
$$

where $\mathbf{I}$ is the $2 \times 2$ identity matrix and $\theta=2 \pi n_{1} L_{1} / \lambda$ is the phase shift within the dielectric of thickness $L_{1}$, and $\mathbf{t}_{1}$ and $\mathbf{t}_{2}$ are transmission matrices. Using $\mathbf{E}_{r}=\mathbf{S}_{11} \mathbf{E}_{i}=\left(\mathbf{T}_{21}\right)^{-1} \mathbf{T}_{11} \mathbf{E}_{i}$, where $\mathbf{S}$ is the scattering matrix, we obtain the reflected field. Note that the $\mathbf{t}$ matrices cancel out of the problem since we are only interested in reflection from the system. When a second nongyrotropic dielectric layer is added, the resulting T-matrix is a product of the T-matrix for a one-layer problem and a propagator

$$
\begin{aligned}
\mathbf{T}^{(2)}= & \left(\mathbf{t}_{1} \mathbf{t}_{2} \mathbf{t}_{3}\right)^{-1}\left[\begin{array}{ll}
\mathbf{I} e^{i \alpha} & \mathbf{r}_{3} e^{-i \alpha} \\
\mathbf{r}_{3} e^{i \alpha} & \mathbf{I} e^{-i \alpha}
\end{array}\right] \\
& \times\left[\begin{array}{ll}
\mathbf{I} e^{i \theta}+\mathbf{r}_{1} \mathbf{r}_{2} e^{-i \theta} & \mathbf{r}_{2} e^{i \theta}+\mathbf{r}_{1} e^{-i \theta} \\
\mathbf{r}_{1} e^{i \theta}+\mathbf{r}_{2} e^{-i \theta} & \mathbf{I} e^{-i \theta}+\mathbf{r}_{1} \mathbf{r}_{2} e^{i \theta}
\end{array}\right],
\end{aligned}
$$

where $\alpha=2 \pi n_{2} L_{2} / \lambda$. The reflection matrices are now (1) and the diagonal matrices $\mathbf{r}_{2}=\left(n_{2}-n_{3}\right) /\left(n_{2}+n_{3}\right) \mathbf{I}$ and $\mathbf{r}_{3}=\left(n_{3}\right.$ $-1) /\left(n_{3}+1\right) \mathbf{I}$. This procedure can be extended easily to the general case of $k$ layers.

Using this formalism, we can evaluate the Kerr rotation from any sequence of dielectric layers. In the following, we analyze the potential of three possible cases to realize Kerr enhancement in nanostructures: single lossless layer, multiple lossless layers, lossless plus highly lossy (metallic) layer. The results are experimentally verified by MOKE measurements on ferromagnetic nickel films.

The simplest way to achieve cavity enhancement of the Kerr rotation is to add a single lossless dielectric layer characterized by a real refractive index $n_{1}$. In this case, $\Phi$ can be calculated analytically ${ }^{11,19}$ or by using the matrix formalism to determine the enhancement factor as a function of $n_{1}$. This is shown in Fig. 2 using nickel as magnetic material and a wavelength of $785 \mathrm{~nm}$ (assuming $n=2.43+4.31 i^{20}$ and a bare Ni Kerr rotation of $0.1^{\circ}$ ). The Kerr rotation can be increased to $90^{\circ}$ for a dielectric index of 4.1. Also shown as circles are the experimental enhancement values $\Phi / \Phi^{(\text {bare Ni) }}$ we found using $\mathrm{SiO}_{2}$ and $\mathrm{SiN}$ layers on nickel. Both maximum enhancement and Kerr rotation as a function of dielectric thickness (inset) show excellent agreement with theory with no free parameters. The analysis suggests that a single layer is sufficient to achieve the desired large Kerr enhancement. However, dielectrics with index on the order of 4 such as $\mathrm{Si}$ or Ge are lossless only at infrared wavelengths. The use of longer wavelength light in near-field optics strongly re-

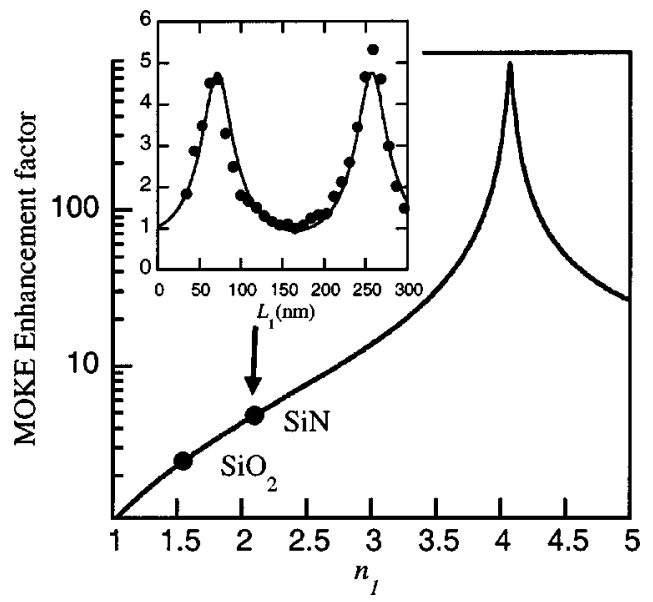

FIG. 2. Cavity enhancement factor of MOKE for a single dielectric layer of index $n_{1}$ on a nickel film at cavity resonance, as predicted by T-matrix theory (-), and measured (-). Inset: Enhancement in SiN coated nickel as a function of dielectric thickness; theory $(-)$ and experiment $(\bullet)$.

duces throughput through a submicron near-field aperture and significantly deteriorates the available signal. A single dielectric layer is therefore not ideal for magneto-optic studies of nanostructures.

The second option to achieve large enhancements is to use multiple layers of lower index dielectrics such as $\mathrm{SiN}$ and $\mathrm{SiO}_{2}$ that are lossless at visible wavelengths. This can be conveniently modeled using the above-described T-matrix approach. We find again that large enhancement factors are possible by optimizing the layer sequence. For example, using six dielectric layers of $\mathrm{Ni}: \mathrm{SiO}_{2}: \mathrm{SiN}: \mathrm{SiO}_{2}$ : $\mathrm{SiN} ; \mathrm{SiO}_{2}: \mathrm{SiN}$, an enhancement factor of 310 is obtained, clearly exceeding the single-layer enhancements of the individual materials shown in Fig. 2. When combined with highresolution Kerr microscopy, however, this presents a problem: the total thickness of this multilayer sequence is $706 \mathrm{~nm}$, which is close to the wavelength and makes true near-field measurements impossible. Thus, in this approach the spatial resolution is compromised.

A third approach is to add a thin, lossy metallic layer on top of a single dielectric layer. Intuitively, this modifies the effective reflectivity of the dielectric-air interface matching better the reflectivity of the dielectric/magnet interface to create a Fabry-Perot cavity with higher $Q$. Using the matrix formalism we found that, in principle, for any metal, a thickness exists that increases the Kerr rotation to $90^{\circ}$ even if silicon dioxide is used as dielectric layer. In order to verify these theoretical findings, we coated several nickel films with a silicon dioxide layer whose thickness was graded across the sample. Gold films with thicknesses between 2.2 and $7 \mathrm{~nm}$ were then deposited on top and the Kerr rotation was measured as a function of both layer thicknesses. The resulting curves as a function of $\mathrm{SiO}_{2}$ thickness are depicted in Fig. 3(a) and the maximum enhancement factor as function of Au thickness is shown in the inset. We find that maximum enhancement is achieved for a gold thickness of $4.1 \mathrm{~nm}$ and that the sign of the Kerr rotation switches as the gold thickness is increased. In order to describe these results with the matrix theory, the microscopic structure of the gold layers needs to be taken into account. AFM images showed thickness variations on the order of $1.5 \mathrm{~nm}$, i.e., $20 \%-68 \%$ the nominal value. Therefore, for a given nominal gold thickness and measured thickness broadening $\Delta L$, the polarization roto AIP license or copyright; see http://apl.aip.org/apl/copyright.jsp 


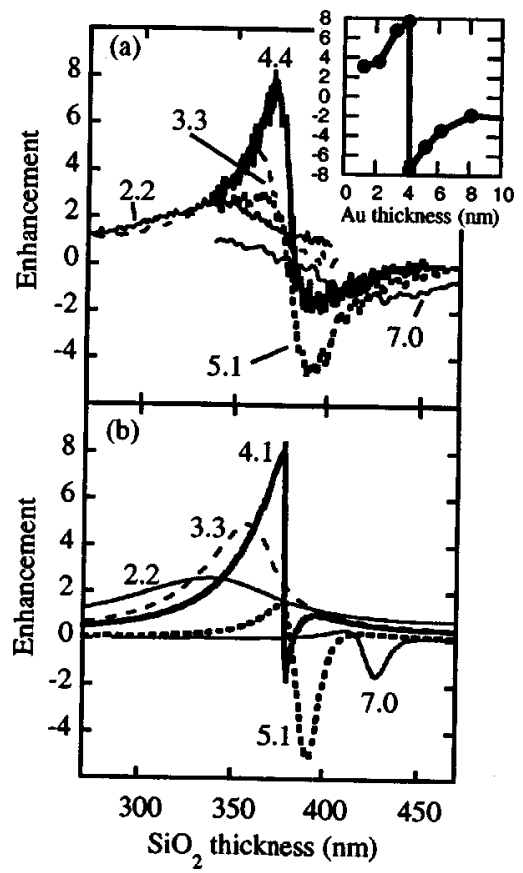

FIG. 3. (a) Measured MOKE enhancement factor of a nickel film coated with silicon dioxide and different thicknesses of gold (labeled in $\mathrm{nm}$ ). Inset: Peak enhancement vs, gold thickness. (b) Fits to two-layer T-matrix theory including grain structure.

tation $\Phi\left(L_{2}\right)$ needs to be convolved with a Gaussian to yield a broadened curve

$$
\Phi_{B}\left(L_{2}\right)=N \int \Phi\left(L_{2}-L^{\prime}\right) e^{-\left(L_{2}-L\right)^{2} / \Delta L^{2}} d L^{\prime}
$$

with appropriate normalization $N$. The real part of the $\mathrm{Au}$ refractive index $n_{2}$ was fixed at $0.08^{20}$ and the imaginary (absorption) part was varied to fit the data and was found to increase with gold thickness in agreement with intuition. After taking the granular structure of the gold film into account, the measured enhancement curves are reproduced very well [Fig. 3(b)]. Both the maximum enhancement limited by the film nonuniformity as well as the sign change and the peak shift with increasing gold thickness agree with the theory. Finally, Fig. 4 shows the enhancement obtained from a nickel film with $\mathrm{SiO}_{2}$ and a $7 \mathrm{~nm}$ gold coating with smaller thickness variations $(\Delta L=1.3 \mathrm{~nm})$. As expected, the enhancement is larger and reaches a value of 16.25 . The data

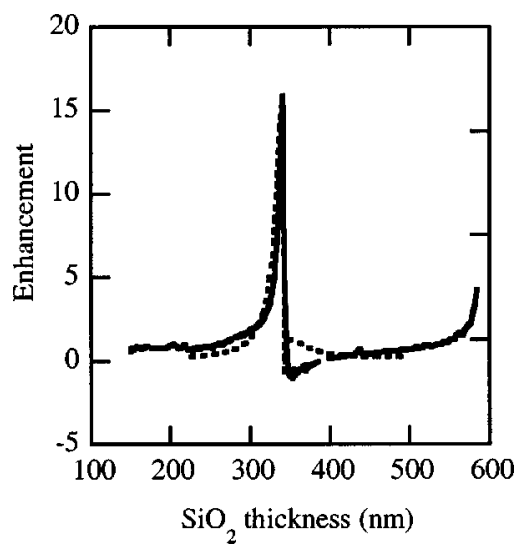

FIG. 4. Measured enhancement for a nickel film coated with silicon dioxide and $7.0 \mathrm{~nm}$ of gold (-) and a fit to T-matrix theory $(\cdots)$ including grain structure. are taken for the cavity's second enhancement peak; the equivalent first peak is at $115 \mathrm{~nm}$, which allows for yet thinner coatings. This lossless dielectric plus thin metal film thus provides the best approach to nanomagnet Kerr microscopy: it allows for a significant increase of the Kerr rotation compared to a single dielectric layer without adding significant additional thickness above the magnetic structure. This enables highly sensitive near-field measurements at visible wavelengths. We point out that the analysis can be readily applied to other combinations of metals and dielectrics and even larger enhancements can be achieved with metals that form a more uniform film. Furthermore, the reduction of reflected intensity at the enhancement maximum has not presented a limitation for the experiments and preliminary studies on microstructured magnets using fiber imaging have shown that the Kerr enhancement indeed improves the available signal-to-noise measurements by an order of magnitude.

In summary, we have investigated enhanced magnetooptic Kerr rotation from a magnetic film using dielectric coatings. For Kerr microscopy of individual single-domain nanomagnets, the polarization rotation needs to be increased while maintaining ultrahigh spatial resolution. Using T-matrix analysis and a series of experiments on nickel films, we found that the best way to achieve this goal is to use a multilayer structure composed of a lossless dielectric and a thin metallic layer. We demonstrated the importance of the surface structure of the metallic film. This approach facilitates noninvasive studies on single-domain nanomagnets and allows for demanding experiments such as time-resolved magnetization switching.

This work was supported by NSF under Grant No. ECS 0245425 .

${ }^{1}$ S. K. Nair and R. M. H. New, IEEE Trans. Magn. 34, 1916 (1998).

${ }^{2}$ G. A. Prinz, Science 282, 1660 (1998).

${ }^{3}$ E. C. Stoner and E. P. Wohlfarth, Philos. Trans. R. Soc. London, Ser. A 220, 599 (1948)

${ }^{4}$ W. F. Brown, Jr., Phys. Rev. 105, 1479 (1957)

${ }^{5}$ H. Schmidt, J. Appl. Phys. 93, 2107 (2003).

${ }^{6}$ F. Ross, H. I. Smith, T. Savas, M. Schattenburg, M. Farhoud, M. Hwang, M. Walsh, M. C. Abraham, and R. J. Ram., J. Vac. Sci. Technol. B 17, 3168 (1999).

${ }^{7}$ S. Sun, C. B. Murray, D. Weller, L. Folks, and A. Moser, Science 287, 1989 (2000)

${ }^{8}$ M. C. Abraham, H. Schmidt, T. A. Savas, H. I. Smith, C. A. Ross, and R. J. Ram, J. Appl. Phys. 89, 5667 (2001).

${ }^{9}$ M. R. Freeman, W. K. Heibert, and A. Stankiewicz, J. Appl. Phys. 83, 6217 (1998).

${ }^{10}$ P. Fumagalli, A. Rosenberger, G. Eggers, A. Munnemann, N. Held, and G. Guntherodt, Appl. Phys. Lett. 22, 2803 (1998).

${ }^{11}$ M. A. Paesler and P. J. Moyer, Near-field Optics: Theory, Instrumentation, and Applications (Wiley, New York, 1996).

${ }^{12}$ A. V. Sokolov, Optical Properties of Metals (Blackie, London, 1967), p. 311.

${ }^{13}$ M. M. Noskov and A. V. Sokolov, Zh. Eksp. Teor. Fiz. 17, 969 (1947).

${ }^{14}$ K. Nakamura, T. Asaka, S. Asari, Y. Ota, and A. Itoh, IEEE Trans. Magn. 21, 165 (1985).

${ }^{15}$ M. Mansuripur, J. Appl. Phys. 67, 6466 (1990).

${ }^{16}$ J. Zak, E. R. Moog, C. Liu, and S. D. Bader, Phys. Rev. B 43, 6423 (1991).

${ }^{17}$ L. A. Coldren and A. Corzine, Diode Lasers and Photonic Integrated Circuits (Wiley, New York, 1995), Chap. 3.

${ }^{18}$ R. Gamble, P. H. Lissberger, and M. R. Parker, IEEE Trans. Magn. 21, 1651 (1985).

${ }^{19}$ N. Qureshi, H. Schmidt, and A. W. Hawkins, IEEE Nano 2003 Proceedings, Vol. 1, pp 175-178.

${ }^{20}$ CRC Handbook of Chemistry and Physics, 83rd Ed. (CRC, Boca Raton, $\mathrm{FL})$. 